\title{
Improved Version of Graph-Cut Algorithm for CT Images of Lung Cancer With Clinical Property Condition
}

\author{
Dr. Samuel Manoharan, \\ Professor, \\ Department of Electronics, \\ Bharathiyar College of Engineering and Technology, \\ Thiruvettakudy, Karaikal, \\ India. \\ Email id: jsamuel@bcetedu.in \\ Prof. Sathish, \\ Department of EEE, \\ Eritrea Institute of Technology, \\ Eritrea. \\ Email id: sathesh4you@gmail.com
}

\begin{abstract}
In a clinical evaluation, the detection of lung cancer is a challenging task. Segmentation methods are used to detect the extra growing nodule. Early diagnosis of lung cancer is significant in clinical research. The early stage of lung nodules is very soft tissues and tough to segment accurately. Generally, conservative graph cut methods are very weak to detect those soft edges in medical images. In this article, the proposed algorithm is improved to obtain the accuracy of the process to segment the edges than the conventional graph cut methods. This investigation is executed to shows the accuracy of lung segmentation.
\end{abstract}

Keywords: lung cancer, computed tomography, graph cut segmentation

\section{INTRODUCTION}

Lung cancer is a deadly disease for humans. Every year, more people are dying due to lung cancer. Lung cancer is more common in western countries like Europe due to cold climatic conditions. Due to the climatic condition, people are addicted to smoking habits but it is rare in young age group people. Cigarette smoking is one of the important reasons for lung cancer.

It is reliable to neglect the habit of smoking. When comparing the risk in the different age groups of people, the severity of developing the disease is very low in youths, medium in the adult age, and very high in the old age peoples. But leaving a smoking habit can reduce the risk of increased lung cancer. After diagnosed with lung cancer, quitting smoking will give better relief mentally and physically [2]. Once smoking is ignored after diagonalizing cancer, the quality of life will be improved.

The accurate segmentation of the nodule is the most challenging task for radiologists. Different segmentation methods have been discussed here. Since there are so many weak edges in medical images, it is difficult to segment the nodule accurately.

The nodule in the lungs due to smoking, age, hemoptysis, and previous malignancy is very difficult to segment from the lungs. Since the medical images are having weak edges, most of the segmentation fails in this case. The primary objective of this research article is to detect and segment the nodule accurately [3].

ISSN: 2582-2012 (online) 
Journal of Artificial Intelligence and Capsule Networks (2020)

Vol.02/ No.04

Pages: 201-206

http://irojournals.com/aicn/

DOI: https://doi.org/10.36548/jaicn.2020.4.002

Lung nodules can easily find on 1 in 500 chest x-ray and 1 in 100 CT scans of the chest approximately. Around 1.5 million people are affected in the United States each year by finding lung nodules [4]. About, fifty percentages of smokers are having nodules in their chest proved by CT scan results.

The presence of the nodule in a lung leads to cancerous (malignant) becomes risky. Also, it completely depends on the age of the person. If the person age is 30 that chance is less than one percent even though having a nodule in their chest which is showed in Table 1. Other factors that increase or decrease the risk that lung nodules become cancer include:

a) Size - Bigger nodules are similar to be malignant than smaller nodules.

b) Smoking - Chain and occasion smokers are more likely to have malignant lung nodules than non-smokers.

c) Occupation - Some occupational exposures raise the probability that a lung nodule is a cancer.

\section{RELATED WORK}

This article presents an improved version of the graph cut to segment the outline of an object with the help of active contours. These active contours can easily deform the object boundary even though there are big discontinuities and abrupt changes of noise. Region of Interest (RoI) data is considered from a certain neighborhood of the previously estimated boundary in each step [5]. The problem statement is framed by many inputs and multilink. And the nearby pixels and nodes are identified by well-organized boundary line operator. The outcome of each iteration is optimal and within the region of interest. This region of interest supports the lively contour to divide the control of estimated boundary and quarantined noise points in the image [6].

Haemoptysis is the medical term for coughing up with blood. There are many causes of haemoptysis, one of which is lung cancer coughing up with blood, otherwise known as haemoptysis, can be very terrifying. In ancient times, lung cancers are misunderstood for many reasons for example are coughing up with blood [8].

Most people do not know the blood comes from the lungs or nose bleeds. Sometimes coughing up with blood arises from the throat. Blood will become foamy appearance after mixed with phlegm [8]. Coughing up with blood doesn't necessarily mean that you are affected by lung cancer. Many reasons can cause you to cough up blood. But since the outlook is better than earlier lung cancer is diagnosed, it is also important to consult a medical physician immediately if you are facing a problem with cough up blood. It is important to make a difference between coughing up with blood (haemoptysis) and blood that is brought up through the mouth from other regions of the body. "Pseudohaemoptysis" describes that sputtering the blood that does not come from lungs or respiratory tubes. Hematemesis is indicating that blood comes from the esophagus and stomach (throwing up blood).

From the year 1999 to 2019, the average age at lung cancer diagnosis was 70 years of age [9]. Table 1 shows the survey for people diagnosed with lung cancer.

Table 1 Survey for Percentages of people diagnosed with lung cancer in stage period

\begin{tabular}{|c|c|c|}
\hline S.NO & AGE PERIOD (In Years) & PERCENTAGE (\%) \\
\hline 1 & under age 20 & 0.1 \\
\hline 2 & 20 and 34 & 0.3 \\
\hline 3 & 35 and 44 & 2.1 \\
\hline 4 & 45 and 54 & 8.7 \\
\hline 5 & 55 and 64 & 21.1 \\
\hline 6 & 65 and 74 & 32.6 \\
\hline 7 & 75 and 84 & 28.2 \\
\hline 8 & 85+ years of age & 6.9 \\
\hline
\end{tabular}

ISSN: 2582-2012 (online)

Submitted: 12.11.2020

Accepted: 03.12.2020

Published: 11.12.2020 
Journal of Artificial Intelligence and Capsule Networks (2020)

Vol.02/ No.04

Pages: 201-206

http://irojournals.com/aicn/

DOI: https://doi.org/10.36548/jaicn.2020.4.002

This article presents a new improved version of graph cut segmentation methods for lung CT scans. Initially, it determines each voxel feature vector that contains intensity, figure index, and 3D spatial location. Generally, the graph cut methods are gathering the pixels in medical images by more energy compaction that includes spatial, intensity features as well as shape [8]. Here, the proposed improved version of the graph cut method has been evaluated by the clinical dataset. Visual inspection of different kinds of injuries, as well as quantitative evaluation, has been done.

Cancer cells are growing faster than the tissue around them to become a tumor. Progressive growth is therefore a central feature of cancer and a critical factor in distinguishing cancerous nodules from benign [11]. There is a characteristic volume doubling time, the interval it takes for a nodule to double in volume. The nodule shape is spherical and it increases in diameter such as $2 \mathrm{~mm}$ which will increase 7 or $9 \mathrm{~mm}$. It shows a doubling of the volume of the nodule [12].

\section{PROPOSED METHOD}

The pre-processing algorithm has written and it is followed by the proposed algorithm for the segmentation.

\section{STEP 1}

1(a) Input image $P_{1}$ (read the original input image)

1(b) Opening: $\mathrm{P}_{1} \Rightarrow>$ eroded image $\mathrm{P}_{2}=>$ dilated image $\mathrm{P}_{3}$ (erode and then dilate the original image to get the Opening image)

1(c) Closing: $\mathrm{P} 1 \Rightarrow$ dilated image $\mathrm{P}_{4}=>$ eroded image $\mathrm{P}_{5}$ (dilate and then erode the original image to get the Opening image)

1(d) Top hat: $\mathrm{P} 6<=\mathrm{P}_{1}-\mathrm{P}_{3}$ (subtract the opening image from the original image)

1(e) Bottom hat: $\mathrm{P} 7<=\mathrm{P}_{5}-\mathrm{P}_{1}$ (subtract the original image from the closing image)

1(f) $\quad \mathrm{P}_{1}+\mathrm{P}_{6} \Leftrightarrow \mathrm{P}_{8}-\mathrm{P}_{7}=>\mathrm{P}_{9}$ (superimpose the original and top hat image and subtract the Bottom hat image from the output image)

$1(\mathrm{~g}) \quad \mathrm{P}_{9}=>$ enhanced image (get the output as enhanced image for preprocessing)

\section{STEP 2}

Enhanced images experiments the proposed segmentation method which generates the region and boundary information. Find out the region and boundary parameter values with aid of the Sobel operator.

\section{STEP 3}

To solve the problem associated with the graph-cut method in terms of $\omega$, new techniques are introduced in this new approach completes precisely in the segmentation and the absence of associate the regularizing parameter [7]. This new approach contains a boundary factor. Due to holding the region information, the conservative graph cut method has more energy function. The new approach reduces energy function during the process. This is shown in equation 7.

3(a) Remove the region parameter from the energy function.

3(b) Calculate the energy function using the boundary parameter.

$$
E_{P(L)}=\sum_{\{p, q\} \in 1 N} B(p, q) \delta\left(L_{p} \neq L_{q}\right) F_{C}(p, q)
$$

Where, $\mathrm{F}_{\mathrm{c}}(p, q)$ specifies consequences of the pixel $\mathrm{p}$ and $\mathrm{q}$ are contained to the same label.

$\mathrm{F}_{\mathrm{c}}(p, q)=\operatorname{prob}\left(\mathrm{IA}_{\mathrm{P}} / \mathrm{A}_{\mathrm{P}=0}\right) \operatorname{prob}\left(\mathrm{IA}_{\mathrm{q}} / \mathrm{A}_{\mathrm{q}=0}\right)+\operatorname{prob}\left(\mathrm{IA}_{\mathrm{P}} / \mathrm{A}_{\mathrm{P}=-1}\right) \operatorname{prob}\left(\mathrm{IAs}_{\mathrm{q}} / \mathrm{A}_{\mathrm{q}=1}\right)$

The object background intensity histograms should have the pixel p probability with that. It shows in the equation wise,

$$
\operatorname{prob}\left(\mathrm{IA}_{\mathrm{P}} / \mathrm{A}_{\mathrm{P}=1}\right) \text { and }\left(\mathrm{IA}_{\mathrm{q}} / \mathrm{A}_{\mathrm{q}=0}\right)
$$

ISSN: 2582-2012 (online) 
Journal of Artificial Intelligence and Capsule Networks (2020)

Vol.02/ No.04

Pages: 201-206

http://irojournals.com/aicn/

DOI: https://doi.org/10.36548/jaicn.2020.4.002

\section{a) Correctness:}

The size of the memory required is reduced even though there is no regional information. This approach can apply the mixture module for color images. When handling the color images, the following model should be appended with the algorithm.

$$
\mathrm{I} A_{P}=\Pi^{T} A_{t}\left(C_{P}\right)
$$

Where, " $\Pi$ " indicates the transformation factor to greyscale.

\section{STEP 4}

Comparing the obtained segmented images graphically with existed methods and proved accurate in the graphical method.

\section{RESULTS AND DISCUSSION}

The comparisons of conventional and improved graph cut segmentation are shown in the below-mentioned images. The following parameters are clinical properties to justify the obtained results [10].

1. Age

2. Smoking

3. Hemoptysis

4. Previous malignancy

5. Month duration

The various nodule sized input $\mathrm{CT}$ scan images are shown in Fig 1, a \& b and the preprocessing has been done for all the images and the enhanced images are shown in Fig 2, a \& b.

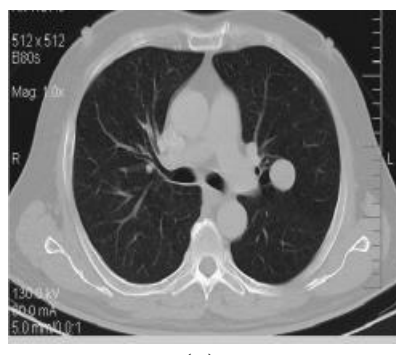

(a)

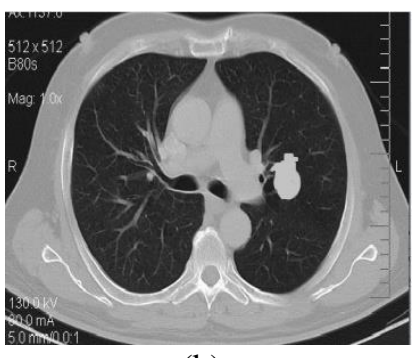

(b)

Fig 1 (a), (b) Original images

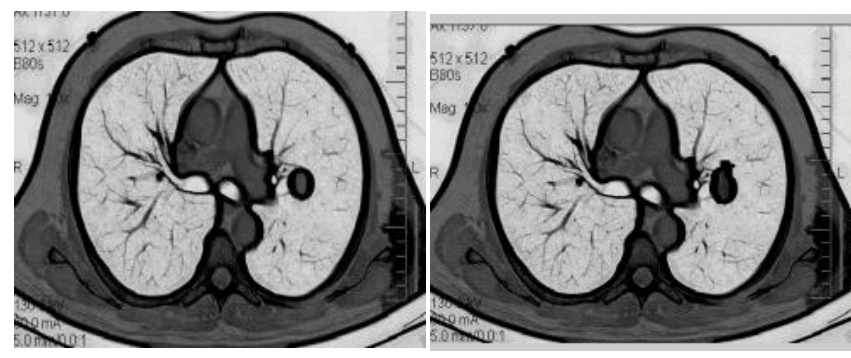

(a)

(b)

Fig 2 (a), (b) Enhanced images

The following figures show the output of different segmentation methods. In figure $3 \mathrm{a} \& \mathrm{~b}$ are the segmented output with aid of the watershed algorithm for enhanced previous images.

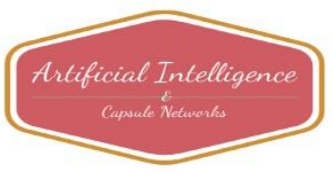


Journal of Artificial Intelligence and Capsule Networks (2020)

Vol.02/ No.04

Pages: 201-206

http://irojournals.com/aicn/

DOI: https://doi.org/10.36548/jaicn.2020.4.002

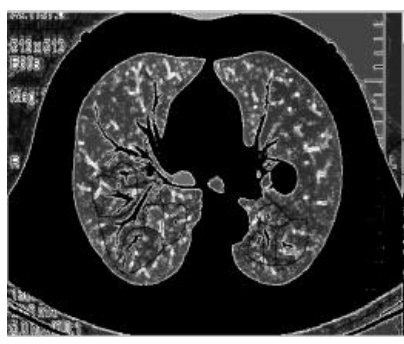

(a)

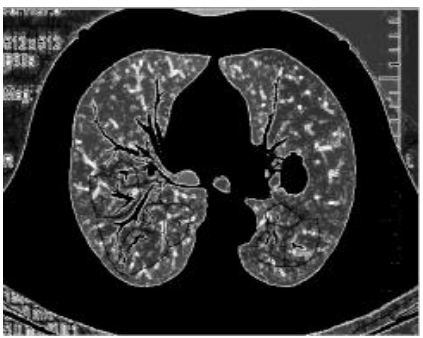

(b)

Fig 3 (a), (b) Watershed segmented images

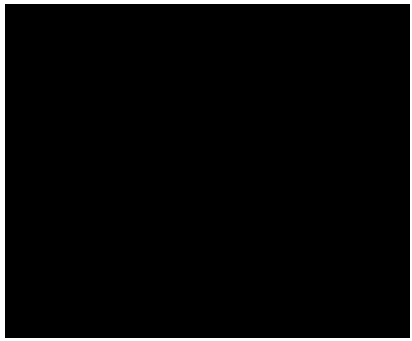

(a)

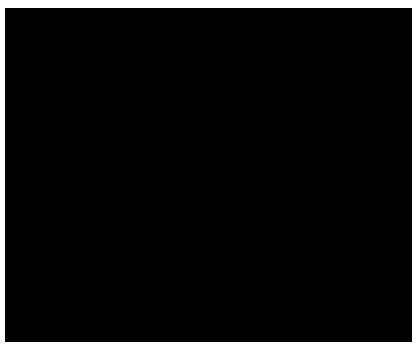

(b)

Fig 4 (a), (b) Graph cut segmented images

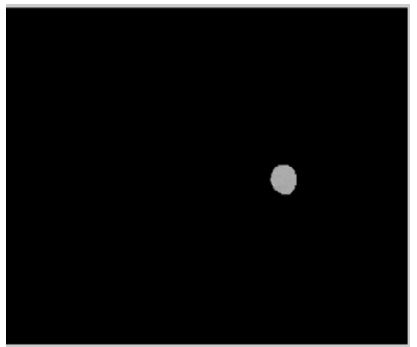

(a)

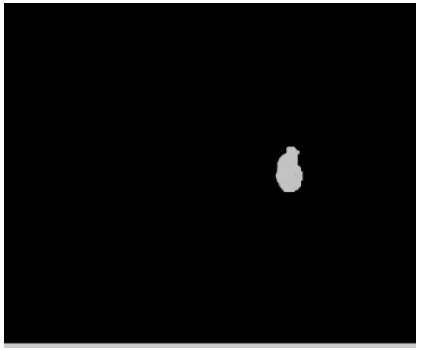

(b)

Fig 5 (a), (b) Improved graph cut segmented images

Figure 5 (a) and (b) shows the results of an improved version of the graph cut segmented from the given image. In the watershed algorithm, over-segmentation will mislead accuracy. The proposed method proves accurate segmentation. This obtained result image is agreed by many medical practitioners based on this accuracy. Then the doctor's prediction becomes easier. From this graphical figures, our proposed techniques show that the results are very accurate agreed by the medical practitioners.

Table 2 Comparison of results between conservative and proposed methods

\begin{tabular}{|c|c|c|c|c|}
\hline S.No & Methods & Energy function & Shape Marked & Accuracy level \\
\hline 1 & Watershed algorithm & More & Yes & Moderate \\
\hline 2 & Graph cut Method & More & No & High \\
\hline 3 & Proposed Method & Moderate & No & High \\
\hline
\end{tabular}

Table 2 shows the improved version of graph cut methods consumes less energy and the accuracy is very high when compared to other conservative methods. While considering the radiographic property the proposed method become excellent in energy function consumption. For the proposed method, the shape is the prior concern for early detection of lung cancer.

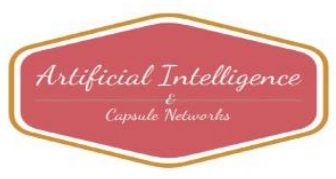


Journal of Artificial Intelligence and Capsule Networks (2020)

Vol.02/ No.04

Pages: 201-206

http://irojournals.com/aicn/

DOI: https://doi.org/10.36548/jaicn.2020.4.002

\section{CONCLUSION}

In the medical field, Lung cancer is a deadly disease for the human sector. Doctors predict the symptoms of lung cancer are due to smoking habits. The nodule plays a major role in diagnosing lung cancer. It is a challenge for radiologists to detect and segment the nodule. So there is a need for an advanced segmentation method since the medical images have so many weak edges.

The image database has been collected from Dr. V. Prabakarakumar MBBS, DMRD consultant diagnostic radiologist and sonologist the best scan center, Coimbatore, India. The images were not clear, so to enhance the contrast pre-processing has been performed. The pre-processed image is undergone with the assistance of watershed segmentation, though the results of watershed segmentation are not satisfied accurately. So our improved segmentation technique is been implemented and the prediction of lung cancer becomes easier for the medical physician. While executing the proposed method, it occupies more memory to process the algorithm. Also, to some extent, the proposed algorithm needs further development in handling the energy function as well as minimize memory storage. The proposed algorithm becomes more efficient while considering the radiographic parameter.

\section{ACKNOWLEDGEMENT}

We would like to thank the Consultant Diagnostic Radiologist \& Sonologist Dr. V. Prabakarakumar MBBS, DMRD of Best Scan Centre Coimbatore for his great support. We would like to extend our thank Ms.Shanmuga Priya who surveyed for this researcharticle.

\section{REFERENCES}

[1] S. Beucher and F. Meyer, "The morphological approach to segmentation: The watershed transform," in Mathematical Morphology in Image Processing, E. R. Dougherty, Ed. New York: Marcel Dekker, 1993, vol. 12, pp. 433-481.

[2] Lan,Shouren; Liu, Xin; Wang, Lisheng "A Visually Guided Framework for Lung Segmentation and Visualization in Chest CT Images" Published in Journal of Medical Imaging and Health Informatics 8(3):485493, March 2018 DOI: 10.1166/jmihi.2018.2325

[3] J. Weickert, "Fast segmentation methods based on partial differential equations and the watershed transform," in Proc. DAGM Symp., 1998, pp. 93-100.

[4] J. B. T. M. Roerdink and A. Meijster, "The watershed transform: Definitions, algorithms and parallelization strategies," Fundamental Informaticae, vol. 41, pp. 187-228, 2000.

[5] S. Armato III, M. Giger, and H. MacMahon, "Automated detection of lung nodules in CT scans: Preliminary result,” Med. Phys. 28, 1552-1561.2001.

[6] Jiyang Dong et al "A Novel Approach of Multiple Objects Segmentation Based on Graph Cut" IEEE Conference on Multimedia Information Processing and Retrieval (MIPR) April 2018, DOI: 10.1109/MIPR.2018.00074

[7] Gradient Based Techniques for the Avoidance of Over-segmentation Conference paper, DOI: 10.13140/2.1.4060.2564, June 2010.

[8] Notter, Robert H," Lung surfactants: basic science and clinical applications "New York, N.Y: Marcel Dekker. pp. 120. ISBN 0-8247-0401-0. Retrieved 2008-10-11.

[9] Bhadauria H S, Dewal M L, "Comparison of Edge Detection Techniques on Noisy abnormal Lung CT Image before and after Using Morphological Filter", 2010.

[10] T.H.Le,et al "Image segmentation based on modified graph-cut algorithm"' Electronics Letters, Vol.46 No.16, 5th September 2010, DOI:10.1049/el.2010.1692.

[11] Shuangfeng Dai et al "A novel approach of lung segmentation on chest CT images using graph cuts" Published in Neurocomputing DOI: 10.1016/j.neucom.2015.05.044, May 2015.

[12] Shuangfeng Dai et al "Lung segmentation with improved graph cuts on chest CT images" published in 3rd IAPR Asian Conference on Pattern Recognition (ACPR),November 2015.

ISSN: 2582-2012 (online) 